DRAFT VERSION MARCH 22, 2022

Preprint typeset using LTEX style emulateapj v. 11/10/09

\title{
ALFVÉN WAVE TURBULENCE AND PERPENDICULAR ION TEMPERATURES IN CORONAL HOLES
}

\author{
BENJAMIN D. G. CHANDRAN \\ Space Science Center and Department of Physics, University of New Hampshire, Durham, NH 03824; benjamin.chandran@unh.edu \\ Draft version March 22, 2022
}

\begin{abstract}
Low-frequency Alfvén-wave turbulence causes ion trajectories to become chaotic, or "stochastic," when the turbulence amplitude is sufficiently large. Stochastic orbits enable ions to absorb energy from the turbulence, increasing the perpendicular ion temperature $T_{\perp \mathrm{i}}$ even when the fluctuation frequencies are too small for a cyclotron resonance to occur. In this paper, an analytic expression for the stochastic heating rate is used in conjunction with an observationally constrained turbulence model to obtain an analytic formula for $T_{\perp \mathrm{i}}$ as a function of heliocentric distance $r$, ion mass, and ion charge in coronal holes at $2 R_{\odot} \lesssim r<15 R_{\odot}$. The resulting temperature profiles provide a good fit to observations of protons and $\mathrm{O}^{+5}$ ions at $2 R_{\odot} \lesssim r \lesssim 3 R_{\odot}$ from the Ultraviolet Coronagraph Spectrometer (UVCS). Stochastic heating also offers a natural explanation for several detailed features of the UVCS observations, including the preferential and anisotropic heating of minor ions, the rapid radial increase in the $\mathrm{O}^{+5}$ temperature between $1.6 R_{\odot}$ and $1.9 R_{\odot}$, and the abrupt flattening of the $\mathrm{O}^{+5}$ temperature profile as $r$ increases above $1.9 R_{\odot}$.
\end{abstract}

Subject headings: solar wind - Sun: corona — turbulence — waves — MHD

\section{INTRODUCTION}

Measurements of ion and electron temperatures show that the solar wind undergoes substantial heating throughout its transit from the solar surface to the heliospheric termination shock. A number of considerations suggest that much of this heating results from Alfvén-wave turbulence, as suggested over forty years ago by Coleman (1968). For example, Alfvén-wave-like fluctuations in the velocity, magnetic field, and electric field are often present in the interplanetary medium, with power spectra that vary approximately as power laws over a broad range of length scales (Belcher \& Davis 1971; Goldstein et al. 1995; Tu \& Marsch 1995; Bruno \& Carbone 2005; Bale et al. 2005). Alfvén waves have also been measured remotely in the chromosphere (De Pontieu et al. 2007) and corona (Tomczyk et al. 2007), and radio observations at heliocentric distances $r$ as small as $\sim 5 R_{\odot}$ reveal a broad spectrum of density fluctuations that are consistent with passive-scalar mixing of entropy modes by Alfvén-wave turbulence (Harmon \& Coles 2005; Chandran et al. 2009). At $r \gtrsim 60 R_{\odot}$, the amplitudes of velocity and magneticfield fluctuations are positively correlated with temperature (Grappin et al. 1990), and the turbulent dissipation rate implied by the measured fluctuation amplitudes is comparable to the heating rate inferred from measurements of the proton and electron temperature profiles (Smith et al. 2001; Cranmer et al. 2009; Stawarz et al. 2009).

On the other hand, it has for some time been unclear whether Alfvén-wave turbulence can explain the ion temperatures and temperature anisotropies observed in the fast solar wind and coronal holes (the open-magnetic-field-line regions from which the fast wind emanates). For example, observations from the Ultraviolet Coronagraph Spectrometer (UVCS) show that minor ions such as $\mathrm{O}^{+5}$ are heated to temperatures greatly exceeding the proton and electron temperatures at $2 R_{\odot} \lesssim r \lesssim 3 R_{\odot}$, with thermal motions that are much more rapid in directions perpendicular to the magnetic field $\mathbf{B}$ than along the magnetic field - i.e., $T_{\perp} \gg T_{\|}$(Kohl et al. 1998; Li et al. 1998; Antonucci et al. 2000). Similarly, in-situ measurements from the Helios and Wind satellites show that $T_{\perp \mathrm{p}}>T_{\| \mathrm{p}}>T_{\mathrm{e}}$ in low- $\beta$ fast-wind streams at $60 R_{\odot} \lesssim r \leq 1 \mathrm{AU}$, where $T_{\perp \mathrm{p}}$ and $T_{\| \mathrm{p}}$ are the perpendicular and parallel proton temperatures, $T_{\mathrm{e}}$ is the electron temperature, and $\beta=8 \pi p / B^{2}$ is the ratio of the plasma pressure $p$ to the magnetic pressure (Phillips \& Gosling 1990; Marsch et al. 2004).

These observations lead to an apparent difficulty for solarwind-heating models based on Alfvén-wave turbulence for the following reasons. In Alfvén-wave turbulence, the energy cascade is anisotropic, transporting energy primarily to small scales measured perpendicular to $\mathbf{B}$ rather than small scales along B (Shebalin et al. 1983; Goldreich \& Sridhar 1995; Ng \& Bhattacharjee 1996; Galtier et al. 2000; Cho et al. 2002). In wavenumber space, energy cascades primarily to larger $k_{\perp}$, and only weakly to larger $k_{\|}$, where $k_{\perp}$ and $k_{\|}$are the components of the wave vector $\mathbf{k}$ perpendicular and parallel to $\mathbf{B}$. At perpendicular scales $\lambda_{\perp}$ of order the proton gyroradius $\rho_{\mathrm{p}}$, the Alfvén-wave cascade transitions to a kineticAlfvén-wave (KAW) cascade (Bale et al. 2005; Howes et al. 2008b; Schekochihin et al. 2009; Sahraoui et al. 2009), and at $\lambda_{\perp} \lesssim \rho_{\mathrm{p}}$ the fluctuations dissipate. The rms amplitude of the magnetic-field fluctuations at $\lambda_{\perp} \lesssim \rho_{\mathrm{p}}$ is $\ll B$, and thus it seems reasonable to assume that the KAW fluctuations damp at the same rate as linear KAWs at the same $\mathbf{k}$. For the $\beta$ values found in coronal holes and the solar wind, linear KAWs undergo significant electron Landau damping (Ouataert 1998; Leamon et al. (1999). However, the KAWs produced by the anisotropic cascade do not undergo ion cyclotron damping, because their frequencies $\omega$ are much less than the proton cyclotron frequency (Cranmer \& van Ballegooijen 2003; Howes et al. 2008a). In addition, when $\beta \ll 1$, the ion thermal speeds are $\ll v_{\mathrm{A}}$, where $v_{\mathrm{A}}$ is the Alfvén speed. Ions are thus unable to satisfy the resonance condition $\omega-k_{\|} v_{\|}=0$ for Landau damping or transit-time damping, where $v_{\|}$is the velocity component parallel to $\mathbf{B}$, because $\omega / k_{\|} \geq v_{\mathrm{A}}$ for KAWs (Quataert 1998; Hollweg 1999). As a consequence, linear damping of KAWs by ions is negligible when $\beta \ll 1$, 
suggesting that low-frequency Alfvén-wave/KAW turbulence results in negligible ion heating in coronal holes and low- $\beta$ fast-wind streams.

A number of studies have gone beyond the framework of linear wave theory to explore the possibility of perpendicular ion heating by low-frequency turbulence in the solar wind (e.g., Dmitruk et al. 2004; Markovskii et al. 2006; Bourouaine et al. 2008; Parashar et al. 2009; Lehe et al. 2009; - see also Drake et al. 2009). Several investigations have shown that low-frequency Alfvén waves and KAWs can indeed cause perpendicular ion heating if the wave amplitudes are sufficiently large (Johnson \& Cheng 2001; Chen et al. 2001; White et al. 2002; Voitenko \& Goossens 2004). This type of heating is some times referred to as "stochastic heating," since it is predicated upon the stochastic ion orbits that arise in the presence of large-amplitude fluctuations in the electric or magnetic field. This paper addresses the question of whether stochastic ion heating can explain the anisotropic ion temperatures that are observed in coronal holes. The analysis is based on the recent studies by Chandran et al. (2010) and Chandran \& Hollweg (2009), which are reviewed in sections 2 and 3 . Stochastic heating in coronal holes and the inner solar wind is then discussed section 4.

\section{STOCHASTIC ION HEATING}

If the amplitudes of Alfvén waves or KAWs with $\lambda_{\perp} \sim \rho_{\mathrm{i}}$ and $\omega<\Omega_{\mathrm{i}}$ are sufficiently large, then ions undergo "stochastic heating," where $\lambda_{\perp}$ is the wavelength measured perpendicular to $\mathbf{B}, \omega$ is the wave frequency, $\rho_{\mathrm{i}}=v_{\perp \mathrm{i}} / \Omega_{\mathrm{i}}$ is the ion gyroradius, $v_{\perp \mathrm{i}}=\sqrt{2 k_{\mathrm{B}} T_{\perp \mathrm{i}} / m_{\mathrm{i}}}$ is the rms value of $v_{\perp}$ (the ion velocity component perpendicular to $\mathbf{B}$ in the local plasma frame), $\Omega_{\mathrm{i}}=q_{\mathrm{i}} B / m_{\mathrm{i}} c$ is the ion cyclotron frequency, and $m_{\mathrm{i}}, q_{\mathrm{i}}$ and $T_{\perp \mathrm{i}}$ are the ion mass, charge, and perpendicular temperature (Johnson \& Cheng 2001; Chen et al. 2001). Chandran et al. (2010) showed that the amplitude threshold for strong stochastic heating when $\beta \lesssim 1$ can be expressed in terms of the quantity

$$
\varepsilon_{\mathrm{i}}=\frac{\delta v_{\mathrm{i}}}{v_{\perp \mathrm{i}}}
$$

where $\delta v_{\mathrm{i}}$ is the rms amplitude of the velocity fluctuation at $\lambda_{\perp} \sim \rho_{\mathrm{i}}$. For Alfvén-wave and KAW fluctuations, $\varepsilon_{\mathrm{i}} \sim q_{\mathrm{i}} \delta \Phi_{\mathrm{i}} / m v_{\perp \mathrm{i}}^{2}$, where $\delta \Phi_{\mathrm{i}}$ is the rms amplitude of the electrostatic-potential fluctuation at $\lambda_{\perp} \sim \rho_{\mathrm{i}}$, and thus $\varepsilon_{\mathrm{i}}$ is roughly the fractional change in an ion's kinetic energy induced by gyroscale fluctuations during the course of a single cyclotron period. If $\varepsilon_{\mathrm{i}}$ is sufficiently small, then a thermal ion's orbit in the plane perpendicular to $\mathbf{B}$ closely approximates a closed circle in some suitably chosen reference frame. In this case, the ion's magnetic moment $\mu=m_{\mathrm{i}} v_{\perp}^{2} / 2 B$ is nearly conserved, and perpendicular ion heating is weak (Kruskal 1962). On the other hand, as $\varepsilon_{\mathrm{i}}$ increases towards unity, a thermal ion's orbit becomes increasingly chaotic, $\mu$ conservation breaks down, and perpendicular ion heating becomes increasingly strong. For protons, $v_{\perp \mathrm{i}} \sim \beta^{1 / 2} v_{\mathrm{A}}, \delta v_{\mathrm{i}} \sim v_{\mathrm{A}} \delta B_{\mathrm{i}} / B$, and $\varepsilon_{\mathrm{i}} \sim \beta^{-1 / 2} \delta B_{\mathrm{i}} / B$, where $\delta B_{\mathrm{i}}$ is the rms amplitude of the magnetic fluctuation at $\lambda_{\perp} \sim \rho_{\mathrm{i}}$. Thus, when $\beta \ll 1$, $\varepsilon_{\mathrm{i}}$ is much greater than $\delta B_{\mathrm{i}} / B$.

Using phenomenological arguments, Chandran et al. (2010) derived an expression (their equation (37)) for the time scale $t_{\mathrm{h}}$ on which stochastic heating doubles an ion's kinetic energy in the absence of cooling:

$$
t_{\mathrm{h}} \simeq \varepsilon_{\mathrm{i}}^{-3} \Omega_{\mathrm{i}}^{-1} \exp \left(\frac{c_{2}}{\varepsilon_{\mathrm{i}}}\right),
$$

where $c_{2}$ is a dimensionless constant. An important consequence of equation (2) is that stochastic heating is inherently self-limiting - as $T_{\perp \mathrm{i}}$ grows, $\varepsilon_{\mathrm{i}}$ decreases, and the stochastic heating rate decreases as a result. Chandran et al. (2010) evaluated $c_{2}$ by simulating test-particle protons interacting with a spectrum of randomly phased Alfvén waves and KAWs in a low- $\beta$ plasma and found that $c_{2}=0.34$. However, they argued that $c_{2}$ is smaller for Alfvén-wave/KAW turbulence than for randomly phased waves, because turbulence produces coherent structures that increase orbit stochasticity (Dmitruk et al. 2004). In the present paper, $c_{2}$ is taken to be the same for all ion species, and the value

$$
c_{2}=0.15
$$

is chosen in order to match UVCS observations of $\mathrm{O}^{+5}$ temperatures, as discussed further in section 4 below. Chandran et al. (2010) also showed that in low- $\beta$ plasmas stochastic heating by low-frequency Alfvén-wave/KAW turbulence primarily increases an ion species' perpendicular temperature $T_{\perp \mathrm{i}}$, rather than its parallel temperature $T_{\| \mathrm{i}}$.

\section{ALFVÉN-WAVE TURBULENCE IN CORONAL HOLES}

Convective motions at the surface of the Sun randomly stir the footpoints of open magnetic field lines, launching lowfrequency Alfvén waves that propagate into coronal holes and then on into the solar wind. Radial variations in the Alfvén speed $v_{\mathrm{A}}(r)$ couple the outward-propagating Alfvén waves to inward-propagating Alfvén waves (Heinemann \& Olbert 1980; Velli 1993; Hollweg \& Isenberg 2007; Cranmer 2010), and nonlinear interactions among counter-propagating waves then cause the wave energy to cascade to small scales and dissipate, heating the ambient plasma (Velli et al. 1989; Matthaeus et al. 1999; Cranmer et al. 2007; Verdini et al. 2010). The value of the correlation length or "outer scale" at the coronal base, denoted $L_{\perp \mathrm{b}}$, is in the present study taken to be $5 \times 10^{3} \mathrm{~km}$, comparable to the spacing of photospheric flux tubes (Spruit 1981). At larger $r$, the value of $L_{\perp}$ is taken to increase in proportion to the radius of a flux tube, so that

$$
L_{\perp}(r)=L_{\perp \mathrm{b}}\left[\frac{B_{0}\left(r_{\mathrm{b}}\right)}{B_{0}(r)}\right]^{1 / 2},
$$

where $B_{0}$ is the background magnetic field strength and $r_{\mathrm{b}} \simeq$ $1 R_{\odot}$ corresponds to the coronal base (i.e., $x=1$ in equation (9) below).

Chandran \& Hollweg (2009) developed an analytical model of Alfvén-wave reflection and turbulent heating based upon a phenomenological treatment of the energy cascade. Using this model, they calculated the radial profiles of the turbulent dissipation rate per unit mass $\Gamma$ and rms wave amplitude at the outer scale, denoted $\delta v_{0}$, extending the previous model of Dmitruk et al. (2002) to account for the solar-wind velocity. For the case that most of the Alfvén-wave energy is at periods of $\sim 1$ hour or longer, Chandran \& Hollweg (2009) found that

$$
\delta v_{0}=\left(\frac{2 \eta^{1 / 4}}{1+\eta^{1 / 2}}\right)\left(\frac{v_{\mathrm{A}}}{v_{\mathrm{Aa}}}\right)^{1 / 2} \delta v_{\mathrm{a}}
$$




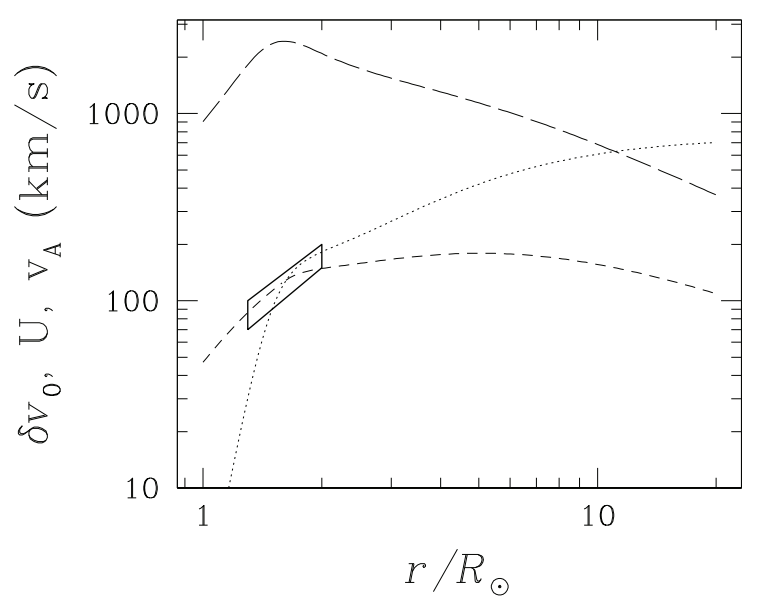

FIG. 1.- The short-dashed line is the rms amplitude of the fluctuating velocity from equation (5) with $\delta v_{\mathrm{a}}=155 \mathrm{~km} / \mathrm{s}$, where $n, B_{0}$, and $U$ are taken from equations $(8)$ through (10). The solid-line box represents the range of non-thermal line widths obtained by Esser et al. (1999). The dotted line is the solar-wind velocity from equation (10), and the long-dashed line is the Alfvén speed from equations 8 and $(9)$.

and

$$
\Gamma=\left(1+\eta^{-1 / 2}\right) \delta v_{0}^{2}\left|\frac{d v_{\mathrm{A}}}{d r}\right|
$$

for $r_{\mathrm{m}}<r \lesssim 20 R_{\odot}$, where $r_{\mathrm{m}}$ is the radius at which the Alfvén speed $v_{\mathrm{A}}=B_{0} / \sqrt{4 \pi n m_{\mathrm{p}}}$ obtains its maximum value, $n$ is the proton number density, $m_{\mathrm{p}}$ is the proton mass, $\delta v_{\mathrm{a}}$ is the value of $\delta v_{0}$ at $r=r_{\mathrm{a}}, r_{\mathrm{a}}$ is the radius of the Alfvén critical point at which the solar-wind outflow speed $U$ equals $v_{\mathrm{A}}, v_{\mathrm{Aa}}$ is the Alfvén speed at $r=r_{\mathrm{a}}$, and

$$
\eta(r)=\frac{n(r)}{n\left(r_{\mathrm{a}}\right)} .
$$

The above results can be applied to coronal holes and the fast solar wind with the use of the following model profiles for $n, B_{0}$, and $U$ :

$$
\begin{gathered}
n=\left(\frac{3.23 \times 10^{8}}{x^{15.6}}+\frac{2.51 \times 10^{6}}{x^{3.76}}+\frac{1.85 \times 10^{5}}{x^{2}}\right) \mathrm{cm}^{-3}, \\
B_{0}=\left[\frac{6}{x^{6}}+\frac{1.5}{x^{2}}\right] \text { Gauss, }
\end{gathered}
$$

and

$$
U=9.25 \times 10^{12} \frac{\tilde{B}}{\tilde{n}} \mathrm{~cm} \mathrm{~s}^{-1},
$$

where $x=r / R_{\odot}, \tilde{B}$ is $B_{0}$ in Gauss, and $\tilde{n}$ is $n$ in units of $\mathrm{cm}^{-3}$. The density in equation (8) is the value from equation (4) of Feldman et al. (1997) plus an additional $r^{-2}$ component chosen to give $n=4 \mathrm{~cm}^{-3}$ at $1 \mathrm{AU}$. Equations (8) through (10) give $r_{a}=11.1 R_{\odot}, r_{m}=1.60 R_{\odot}$, and $U(1 \mathrm{AU})=750 \mathrm{~km} \mathrm{~s}^{-1}$, and lead to the $U$ and $v_{\mathrm{A}}$ profiles shown in figure 1 . The value of $\delta v_{0}$ from equation (5) is also plotted in figure 1, where the value $\delta v_{\mathrm{a}}=155 \mathrm{~km} / \mathrm{s}$ has been chosen so that $\delta v_{0}$ remains bounded by the range of non-thermal line widths obtained by Esser et al. (1999) multiplied by $\sqrt{2}$ to convert from an rms line-of-sight velocity to an rms velocity in the plane perpendicular to $\mathrm{B}$.
In Alfvén-wave turbulence, fluctuation energy cascades from $\lambda_{\perp} \sim L_{\perp}$ to smaller $\lambda_{\perp}$ and then dissipates at $\lambda_{\perp} \lesssim \rho_{\mathrm{p}}$ due to some combination of ion and electron damping. It is assumed that dissipation is negligible at $\lambda_{\perp}>2 \rho_{\mathrm{p}}$, and that

$$
\delta v_{\lambda_{\perp}}=\delta v_{0}\left(\frac{\lambda_{\perp}}{L_{\perp}}\right)^{a}
$$

at $2 \rho_{\mathrm{p}}<\lambda_{\perp}<L_{\perp}$, where $\delta v_{\lambda_{\perp}}$ is the rms amplitude of the fluctuating velocity at perpendicular scale $\lambda_{\perp}$. The constant $a$ is related to the velocity power spectrum $P_{\mathrm{v}}\left(k_{\perp}\right)$. If $P_{\mathrm{V}} \propto k_{\perp}^{-\sigma}$ for $L_{\perp}^{-1} \lesssim k_{\perp} \lesssim\left(2 \rho_{\mathrm{p}}\right)^{-1}$, then $a=(\sigma-1) / 2$. Observations of solar-wind velocity fluctuations at $1 \mathrm{AU}$ find that $\sigma=3 / 2$ (Podesta et al. 2007). Numerical simulations of magnetohydrodynamic (MHD) turbulence generally find that $\sigma=5 / 3$ when $\delta v_{0} \sim v_{\mathrm{A}}$ (Cho \& Vishniac 2000; Müller \& Biskamp 2000; Haugen et al. 2004) and $\sigma=3 / 2$ when $\delta v_{0} \leqslant 0.2 v_{\mathrm{A}}$ (Maron \& Goldreich 2001; Müller \& Grappin 2005; Boldyrev 2006; Mason et al. 2008; Perez \& Boldyrev 2008, 2009). From figure1, $\delta v_{0} / v_{\mathrm{A}}<0.3$ at $r<15 R_{\odot}$, indicating that this near-Sun region is better described by simulations with $\delta v_{0} \lesssim 0.2 v_{\mathrm{A}}$ than simulations with $\delta v_{0} \sim v_{\mathrm{A}}$. Based on the above studies, it is assumed that $\sigma=3 / 2$ and

$$
a=0.25
$$

at $r \leq 15 R_{\odot}$, the region on which this study focuses.

UVCS observations show that $\mathrm{O}^{+5}$ has a larger thermal speed than protons at $r \sim 2 R_{\odot}$, and hence a gyroradius that is several times larger than $\rho_{\mathrm{p}}$. Based in part on this observation, it is assumed that $\rho_{\mathrm{i}}>2 \rho_{\mathrm{p}}$ for minor ions and alpha particles at $r \gtrsim 2 R_{\odot}$. Equation (11) then gives

$$
\delta v_{\mathrm{i}}=\alpha_{\mathrm{i}} \delta v_{0}\left(\frac{\rho_{\mathrm{i}}}{L_{\perp}}\right)^{a}
$$

with

$$
\alpha_{\mathrm{i}}=1 \quad \text { (for } \mathrm{He}^{++} \text {and minor ions). }
$$

In the case of protons, electron Landau damping and stochastic proton heating drain energy from the cascade at $\lambda_{\perp} \sim \rho_{\mathrm{p}}$, reducing $\delta v_{\lambda_{\perp}}$ at $\lambda_{\perp} \sim \rho_{\mathrm{p}}$ relative to the scaling in equation (11). To account for this, it is assumed that

$$
\alpha_{i}=0.71 \quad \text { (for protons), }
$$

where the particular value in equation (15) is chosen so that the results in section 4 match observations of $T_{\perp \mathrm{p}}$ in coronal holes, as described further in the discussion of figure 3. Equations (1) and (13) imply that

$$
\varepsilon_{\mathrm{i}}=\alpha_{\mathrm{i}}\left(\frac{\delta v_{0}}{v_{\perp \mathrm{i}}}\right)^{1-a}\left(\frac{\delta v_{0}}{L_{\perp} \Omega_{\mathrm{i}}}\right)^{a} .
$$

Equation (16) shows that as $T_{\perp \mathrm{i}}$ decreases, $\varepsilon_{\mathrm{i}}$ increases, which in turn increases the stochastic heating rate. One way of understanding this is that Alfvén-wave/KAW fluctuations with $\lambda_{\perp} \sim \rho_{\mathrm{i}}$ cause the electrostatic potential $\Phi$ to vary in a complicated way in the plane perpendicular to $\mathbf{B}_{0}$, with an rms variation of $\delta \Phi_{\mathrm{i}} \sim \rho_{\mathrm{i}} \delta E_{\mathrm{i}}$ over a distance $\rho_{\mathrm{i}}$, where $\delta E_{\mathrm{i}} \sim \delta v_{\mathrm{i}} B_{0} / c$ is the rms amplitude of the electric-field fluctuation at $\lambda_{\perp}=\rho_{\mathrm{i}}$. (The larger but smoother spatial variations in $\Phi$ associated with Alfvén-wave fluctuations at $\lambda_{\perp} \gg \rho_{\mathrm{i}}$ are ignored here, as they lead to drift motion rather than stochastic orbits.) Equation (13) then gives $\delta \Phi_{\mathrm{i}} \propto v_{\perp \mathrm{i}}^{1+a}$, and $\delta \Phi_{\mathrm{i}} / v_{\perp \mathrm{i}}^{2} \propto v_{\perp \mathrm{i}}^{a-1}$. For $a<1$, decreasing $v_{\perp \mathrm{i}}$ causes $q_{\mathrm{i}} \delta \Phi_{\mathrm{i}}$ to become an increasingly 
large fraction of the ions' perpendicular kinetic energy, and the ions' motion in the plane perpendicular to $\mathbf{B}_{0}$ becomes increasingly chaotic as a result.

A possible objection to setting $\rho_{\mathrm{i}}>2 \rho_{\mathrm{p}}$ at $r \gtrsim 2 R_{\odot}$ for $\mathrm{He}^{++}$and minor ions is that preferential heating of heavy ions is assumed from the outset, leaving open the question of how such ions first reach temperatures exceeding $T_{\perp \mathrm{p}}$. This initial evolution can be understood by considering $\mathrm{He}^{++}, \mathrm{O}^{+5}$, and $\mathrm{Fe}^{+11}$ ions with temperatures $\sim T_{\perp \mathrm{p}}$ at small $r$. Given their charge-to-mass ratios, these ions have gyroradii that are comparable to $\rho_{\mathrm{p}}$ when $T_{\perp \mathrm{i}} \sim T_{\perp \mathrm{p}}$, and hence values of $\delta v_{\mathrm{i}}$ that are comparable to the proton value. As a result, the slower, heavier ions have larger $\varepsilon_{\mathrm{i}}$ and much larger stochastic heating rates than protons when $T_{\perp \mathrm{i}} \sim T_{\perp \mathrm{p}}$. These larger heating rates then lead to $T_{\perp \mathrm{i}} \gg T_{\perp \mathrm{p}}$ at larger $r$.

In the Cluster measurements at $1 \mathrm{AU}$ analyzed by Bale et al. (2005), the electric-field power spectrum is slightly larger at $k_{\perp} \rho_{\mathrm{p}}=1$ than one would expect from an extrapolation of the power-law scaling that is present at smaller $k_{\perp}$. The electric-field spectrum is a good proxy for the spectrum of (electron) velocity fluctuations associated with AW/KAW turbulence (Schekochihin et al. 2009), and thus $\alpha_{i}>1$ for protons in this data set. Nevertheless, the value $\alpha_{i}=$ 0.71 is reasonable for protons in coronal holes for two reasons. First, AW/KAW turbulence is more "imbalanced" in coronal holes than at $1 \mathrm{AU}$, with a greater excess of outward-propagating waves over inward propagating waves Cranmer \& van Ballegooijen 2005; Verdini \& Velli 2007). Such imbalance weakens the cascade rate, causing the power spectrum to decrease more rapidly with increasing $k_{\perp}$ near $k_{\perp} \rho_{\mathrm{p}}=1$ in response to a fixed KAW damping rate. Second, the linear damping rate of KAWs at $k_{\perp} \rho_{\mathrm{p}}=1$ is significantly larger in the $\beta \ll 1$ conditions of coronal holes than in the typical $\beta \sim 0.5-1$ conditions found at $1 \mathrm{AU}$ (see, e.g., figure 2 of Howes et al. (2008a)).

\section{ION TEMPERATURE PROFILES}

As described in the introduction, linear damping of KAWs by ions is extremely weak when $\beta \ll 1$ (Quataert 1998). Based in part on this finding, it is assumed in this section that stochastic heating is the primary ion heating mechanism in coronal holes. The $T_{\perp \text { i }}$ profiles that result from this assumption are then calculated, and it is shown that for plausible parameter values the resulting profiles provide a good fit to the observations.

At $r \gtrsim 2 R_{\odot}$, collisional energy exchange between particle species can be neglected to a reasonable approximation, because the energy exchange time scale exceeds the expansion time scale (Esser et al. 1999),

$$
t_{\exp }=\frac{r}{U_{\mathrm{i}}}
$$

The time scale $t_{\text {cond }}$ on which ion temperatures are modified by ion thermal conduction is $\gtrsim r / v_{\| i}$, where this lower limit corresponds to ions streaming freely at their parallel thermal velocity $v_{\| \mathrm{i}}=\sqrt{k_{\mathrm{B}} T_{\| \mathrm{i}} / m_{\mathrm{i}}}$. At $r \gtrsim 2 R_{\odot}$, ion flows are supersonic (Kohl, J., et al. 1998), and thus $t_{\text {cond }}>t_{\exp }$ even in the free-streaming limit. Ion thermal conduction can thus be neglected at $r \gtrsim 2 R_{\odot}$ to a first approximation.

Because collisions and conduction are weak, minor ions at $r \gtrsim 2 R_{\odot}$ evolve towards a state in which

$$
t_{\mathrm{h}} \sim t_{\mathrm{exp}}
$$

for the following reasons. Minor ions draw very little power from the turbulence, and the power spectrum of the turbulence is essentially independent of the minor-ion heating rate $Q_{i}$. Equations (11) and (2) thus imply that $Q_{\mathrm{i}}$ is a highly sensitive function of the minor-ion temperature, with strong heating at small $T_{\perp \mathrm{i}}$ and exponentially weak heating at sufficiently large $T_{\perp \mathrm{i}}$. If $t_{\mathrm{h}} \gg t_{\mathrm{exp}}$ at some radius $r_{1}$, then adiabatic cooling would cause $T_{\perp \mathrm{i}}$ to decrease in proportion to $B_{0}$, and $d \ln T_{\perp \mathrm{i}} / d \ln r$ would be $\leq-2$ near $r_{1}$. The radial decrease in $T_{\perp \mathrm{i}}$ near $r_{1}$ would lead to a sharp decrease in $t_{\mathrm{h}}$, and at larger $r$ the minor ions would leave the adiabatic regime. Conversely, if $t_{\mathrm{h}} \ll t_{\text {exp }}$ at some radius $r_{2}$, then $d \ln T_{\perp \mathrm{i}} / d \ln r \gg 1$ near $r_{2}$. The radial increase in $T_{\perp \mathrm{i}}$ would cause $t_{\mathrm{h}}$ to increase rapidly with increasing $r$, and at larger $r$ the ions would again approach a state in which $t_{\mathrm{h}} \sim t_{\mathrm{exp}}$.

The observed $T_{\perp \mathrm{i}}$ profile of $\mathrm{O}^{+5}$ ions in coronal holes (see figure 3 below) appears to provide an example of the second case just described, in which ions swiftly evolve from a state in which $t_{\mathrm{h}} \ll t_{\exp }$ to a state in which $t_{\mathrm{h}} \sim t_{\mathrm{exp}}$. The $\mathrm{O}^{+5}$ temperature is $<10^{7} \mathrm{~K}$ at $r \leq 1.6 R_{\odot}$, presumably due to collisions with protons. As $\mathrm{O}^{+5}$ ions flow outward past $r=1.6 R_{\odot}$, their temperature increases rapidly to $\sim 10^{8} \mathrm{~K}$ at $r=1.9 R_{\odot}$, where collisional energy exchange with protons is weak. At $r=1.9 R_{\odot}$, the $\mathrm{O}^{+5}$ temperature profile abruptly flattens, with $T_{\perp \mathrm{i}}$ remaining fairly constant out to $2.7 R_{\odot}$, indicating that $t_{\mathrm{h}} \sim t_{\exp }$ at $r \gtrsim 2 R_{\odot}$. These rapid radial variations in $T_{\perp \mathrm{i}}$ and $d T_{\perp \mathrm{i}} / d r$ present a challenge for theoretical models, but can be naturally explained in terms of stochastic heating - stochastic heating of an initially cool minor-ion population quickly increases $T_{\perp \mathrm{i}}$, but then saturates at large $T_{\perp \mathrm{i}}$ because of the reduction in orbit stochasticity $\square$

The strong dependence of $t_{\mathrm{h}}$ on $\varepsilon_{\mathrm{i}}$ implies that $t_{\mathrm{h}} \sim t_{\mathrm{exp}}$ only within a narrow interval of $\varepsilon_{\mathrm{i}}$ values. The midpoint of this interval can be found by equating $t_{\mathrm{h}}$ and $t_{\mathrm{exp}}$, which yields

$$
\varepsilon_{\mathrm{i}}^{-3} \exp \left(\frac{c_{2}}{\varepsilon_{\mathrm{i}}}\right)=\frac{\Omega_{\mathrm{i}} r}{U_{\mathrm{i}}} .
$$

The right-hand side of this equation is $\gg 1$ in coronal holes, leading to values of $\varepsilon_{\mathrm{i}}$ that are $\ll 1$. For example, $\Omega_{\mathrm{i}} r / U_{\mathrm{i}}=$ $6.13 \times 10^{6}$ for $\mathrm{O}^{+5}$ ions at $r=2 R_{\odot}$ given the assumptions listed in the caption of figure 2. Equation (19) then gives $\varepsilon_{\mathrm{i}}=$ $2.96 \times 10^{-2}$, assuming $c_{2}=0.15$. For such small values of $\varepsilon_{i}$, the value of $\varepsilon_{\mathrm{i}}$ becomes relatively insensitive to changes in the right-hand side of equation (19). For example, if $\Omega_{\mathrm{i}} r / U_{\mathrm{i}}$ is increased from $6.13 \times 10^{6}$ by $50 \%$, the resulting decrease in $\varepsilon_{\mathrm{i}}$ is only $5 \%$.

For protons, the comparatively flat $T_{\perp \mathrm{i}}$ profiles seen in UVCS observations (Kohl et al. 1998) rule out the possibility that $t_{\mathrm{h}} \ll t_{\exp }$ or $t_{\mathrm{h}} \gg t_{\exp }$, assuming stochastic heating is the dominant heating mechanism. Thus, $t_{\mathrm{h}} \sim t_{\exp }$ and protons approximately satisfy equation (19). However, protons can attain the required value of $\varepsilon_{i}$ not only by getting hotter or cooler, but also by absorbing energy from the turbulence and reducing the value of $\delta v_{\lambda_{\perp}}$ at $\lambda_{\perp} \sim \rho_{\mathrm{p}}$. No attempt is

1 It should be noted that the observed $\mathrm{O}^{+5}$ temperature profile has also been approximately reproduced by models invoking resonant cyclotron heating by high-frequency Alfvén/ion-cyclotron waves - see, e.g., Cranmer et al. (1999) and Isenberg \& Vasquez (2009). 


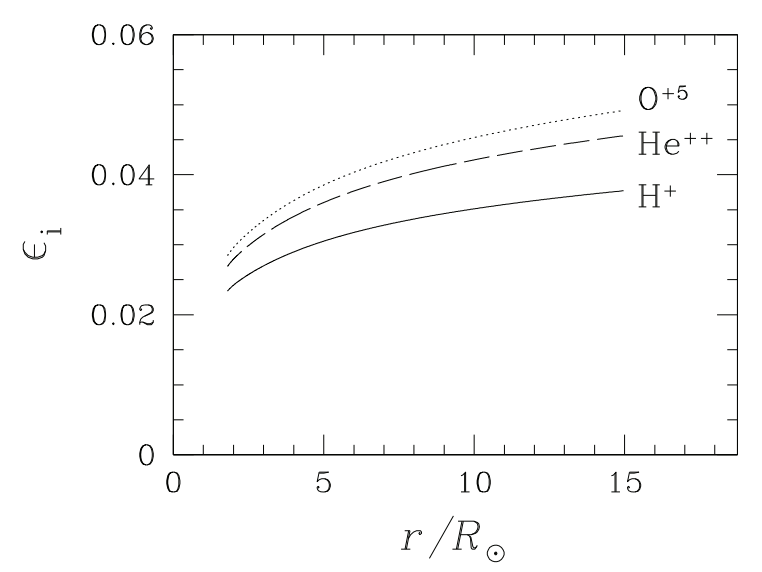

FIG. 2.- The values of $\varepsilon_{\mathrm{i}}$ from equation 19 for protons, alpha particles, and $\mathrm{O}^{+5}$ ions, where $c_{2}=0.15$ and $\Omega_{\mathrm{i}}(r)$ is calculated using equation 9 . For protons, $U_{\mathrm{i}}$ in equation (19) is set equal to the value of $U$ in equation (10). For $\mathrm{He}^{++}$and $\mathrm{O}^{+5}, U_{\mathrm{i}}$ is set equal to $1.75 \mathrm{U}$.

made in this paper to treat the coupled evolution of protons and gyro-scale KAW fluctuations self-consistently. Instead, proton damping (and electron Landau damping) of fluctuations at $\lambda_{\perp} \sim \rho_{\mathrm{p}}$ is modeled simplistically by setting $\alpha_{\mathrm{i}}=0.71$ for protons in equation (15). This particular value is chosen to match the UVCS observations shown in figure 3, as described further below. Although helium comprises only $~ 5 \%$ of the ions in the fast solar wind (Bame et al. 1977), alpha particles are hotter than protons in the fast wind, and may also drain a significant amount of power from the cascade (Marsch et al. 1982; Kasper et al. 2007) [2 The back reaction of helium heating upon the turbulence, however, is neglected in this paper.

To determine $\varepsilon_{\mathrm{i}}$ from equation (19), $B_{0}$ is taken from equation (9) and $U_{\mathrm{i}}$ for protons is set equal to $U$ in equation (10). For other ion species, $U_{\mathrm{i}}$ is taken to be 1.75 times the proton speed, which is consistent with UVCS observations of protons and $\mathrm{O}^{+5}$ ions at $r=3 R_{\odot}$ (Kohl et al. 1998), but is only a rough approximation for other ion species and at other radii. The resulting values of $\varepsilon_{\mathrm{i}}$ for protons, alpha particles, and $\mathrm{O}^{+5}$ ions are shown in figure 2 for $1.8 R_{\odot}<r<15 R_{\odot}$. Since ion thermal conduction and collisional energy exchange between particle species are neglected, the curves are not extended to smaller $r$ where these processes become important.

Once $\varepsilon_{\mathrm{i}}(r)$ is determined using equation (19), $T_{\perp \mathrm{i}}$ can be determined from equation (16), which can be re-written as

$$
T_{\perp \mathrm{i}}=\frac{m_{\mathrm{i}}}{2 k_{\mathrm{B}}}\left[\frac{\alpha_{\mathrm{i}} \delta v_{0}}{\varepsilon_{\mathrm{i}}\left(L_{\perp} \Omega_{\mathrm{i}}\right)^{a}}\right]^{2 /(1-a)} .
$$

As illustrated in figure 2, equation (19) leads to similar values of $\varepsilon_{\mathrm{i}}$ for alpha particles and minor ions. If $\varepsilon_{\mathrm{i}}$ and $\alpha_{\mathrm{i}}$ are the same for some set of ion species (or if $\alpha_{\mathrm{i}} / \varepsilon_{\mathrm{i}}$ is the same), then equation (20) implies that

$$
T_{\perp \mathrm{i}} \propto A\left(\frac{A}{Z}\right)^{l}
$$

for these ions, where $A=m_{\mathrm{i}} / m_{\mathrm{p}}, Z=q_{\mathrm{i}} / e, e$ is the proton

\footnotetext{
${ }^{2}$ For example, the Helium heating rate is comparable to the proton heating rate for the temperature profiles in the right panel of figure 3 given the assumed values of $U_{\mathrm{i}}$.
}

charge, and $l=2 a /(1-a)$. The value of $l$ can also be expressed as

$$
l=\frac{2 \sigma-2}{3-\sigma},
$$

where $\sigma$ is the spectral index of the velocity power spectrum defined in section 2. In this paper, it is assumed that $a=1 / 4$ and $\sigma=3 / 2$, which gives $l=2 / 3$. If instead $a=1 / 3$ and $\sigma=5 / 3$, then $l=1$.

The largest radius at which equations (19) and (20) can be applied is determined by the condition that the parallel thermal speed $v_{\| \mathrm{i}}$ remain $\ll v_{\mathrm{A}}$ so that ion Landau damping and transit-time damping of KAWs can be neglected. Assuming that $n_{0}$ and $B_{0}$ are given by equations $(8)$ and $(9)$, that $T_{\| \mathrm{i}}(r)$ for protons is no greater than the $r$-dependent (isotropic) temperature in equation (47) of Cranmer \& van Ballegooijen (2005), and that protons are the ion species with the largest value of $v_{\| \mathrm{i}}$, one finds that $v_{\| \mathrm{i}}<v_{\mathrm{A}} / 3$ for all ion species at to at least $15 R_{\odot}$.

Temperature profiles from equations (19) and (20) are plotted in figure 3. The left panel of this figure shows $T_{\perp \mathrm{i}}$ for protons and $\mathrm{O}^{+5}$ ions at $1.8 R_{\odot}<r<3.3 R_{\odot}$, and the right panel shows the $T_{\perp \mathrm{i}}$ profiles for four ion species out to $15 R_{\odot}$. The data points in both panels represent the ion kinetic temperatures obtained by Esser et al. (1999) from UVCS observations. The model temperature profiles in this figure were constructed using the $n, B_{0}$, and $U$ profiles in equations (8) through (10) and the values of $L_{\perp}$ and $\delta v_{0}$ given in equations (4) and (5) with $\delta v_{\mathrm{a}}=155 \mathrm{~km} / \mathrm{s}$ as in figure 1 The value $c_{2}=0.15$ was chosen to match the observed $\mathrm{O}^{+5}$ temperatures at $r>1.8 R_{\odot}$. The value $\alpha_{\mathrm{p}}=0.71$ was then chosen to match the observed proton temperatures at $r>1.8 R_{\odot}$.

Possible sources of error in figure 3 include uncertainties in the values of $c_{2}, a, \alpha_{\mathrm{i}}$, and $U_{\mathrm{i}}(r)$. For example, if $c_{2}$ is increased from 0.15 to 0.34 , but all other model parameters are held fixed, then $T_{\perp \mathrm{p}}$ decreases by a factor of 4.6 at $r=2 R_{\odot}$. If $a$ is increased from $1 / 4$ to $1 / 3$ but all other parameters are fixed at their original values, then $T_{\perp p}$ decreases by a factor of 27 at $r=2 R_{\odot}$. If $\alpha_{\mathrm{i}}$ is increased from 0.71 to 1 for protons, then $T_{\perp \mathrm{p}}$ increases by a factor of 2.5 at $r=2 R_{\odot}$. The value of $U_{\mathrm{i}}(r)$ has less of an impact on the ion temperatures that follow from equations (19) and (20). If $U_{\mathrm{i}}$ is decreased from $1.75 U$ to $1.5 U$ for $\mathrm{O}^{+5}$ ions, then $T_{\perp \mathrm{i}}$ increases by only $5.2 \%$ at $r=2 R_{\odot}$.

There is, however, a potentially larger source of error associated with $U_{\mathrm{i}}(r)$. As the relative velocity $\Delta U_{\mathrm{i}}$ between heavy ions and protons approaches $v_{\mathrm{A}}$, the electric field in the heavy-ion frame decreases, since most of the Alfvénwave fluctuations propagate away from the Sun in the proton frame. As a result, the stochastic heating rate $Q_{\mathrm{i}}$ for heavy ions decreases (Chandran et al. 2010). The acceleration of heavy ions to relative flow speeds $\sim v_{\text {A }}$ provides a second possible mechanism (in addition to the reduction of $\varepsilon_{i}$ through the increase of $T_{\perp \mathrm{i}}$ ) for saturating stochastic heating, provided the amplitudes of sunward-propagating waves are much less than the amplitudes of anti-Sunward waves at $\lambda_{\perp} \sim \rho_{\mathrm{i}}$. The effects of $\Delta U_{\mathrm{i}}$ on $Q_{\perp \mathrm{i}}$ are neglected in figure 3 . which is justified at $r \leqslant 3 R_{\odot}$, where UVCS observations show that $\Delta U_{\mathrm{i}} \ll v_{\mathrm{A}}$ (Kohl, J., et al. 1998). However, for the velocity profiles assumed in the construction of figure 3, $\Delta U_{\mathrm{i}} \sim v_{\mathrm{A}}$ near $r=15 R_{\odot}$, suggesting that the heavy ion temperatures near $r=15 R_{\odot}$ may be over-estimated by equation (20).

A self-consistency check on the assumed value of $\alpha_{i}$ for 

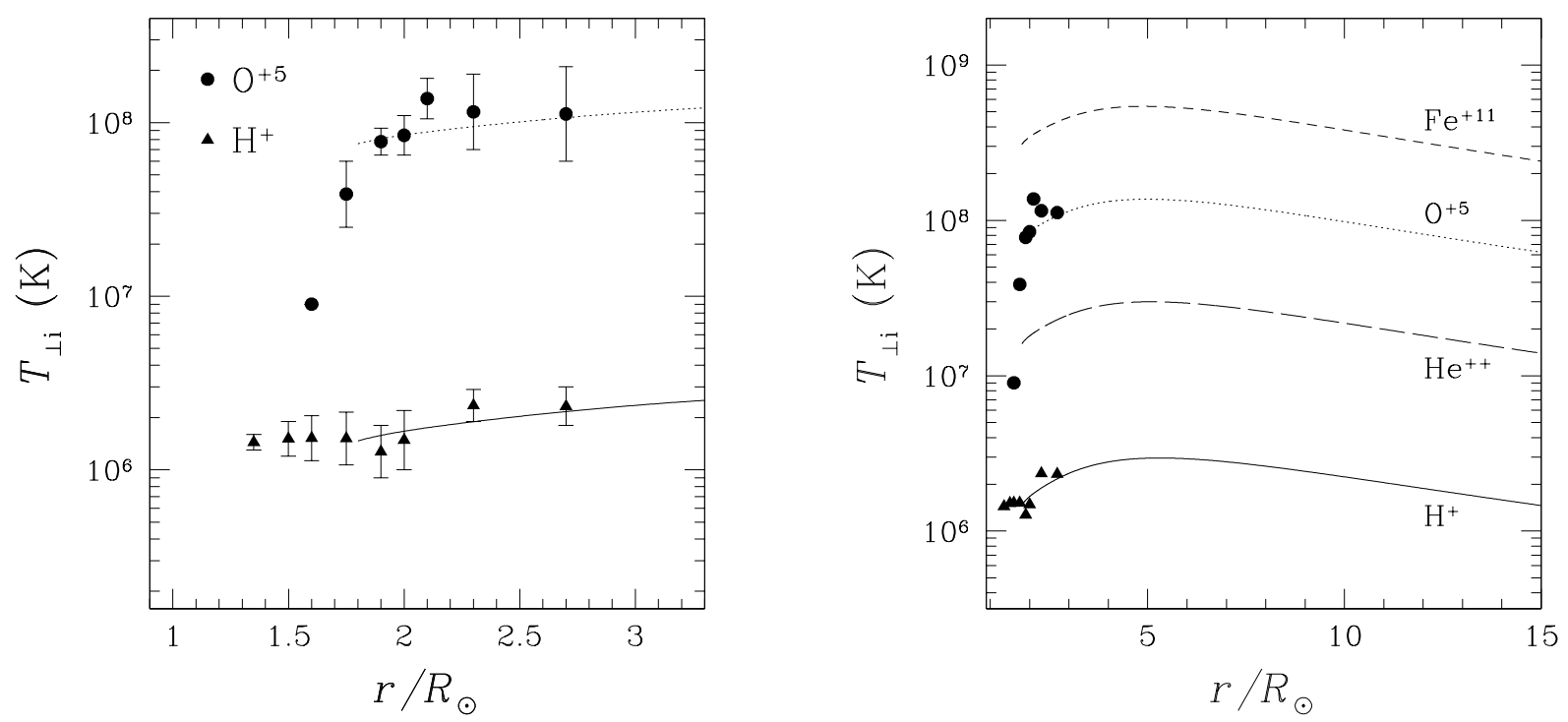

FIG. 3. Left panel: the solid (dotted) line is $T_{\perp \mathrm{i}}$ for protons $\left(\mathrm{O}^{+5}\right)$ from equations 19 and 20. Right panel: the different curves show $T_{\perp \mathrm{i}}$ from equations 19 and (20) for four ion species over a larger range in $r$. Both panels: equations 19 and (20) are evaluated with $\alpha_{\mathrm{i}}=0.71$ for protons and $\alpha_{\mathrm{i}}=1$ for other ions. The value of $U_{\mathrm{i}}$ is set equal to $U$ in equation (10) for protons and $1.75 U$ for other ions. For all curves, $c_{2}=0.15$ and $a=0.25, L_{\perp}$ and $\delta v_{0}$ are taken from equations 4 ) and (5) with $\delta v_{\mathrm{a}}=155 \mathrm{~km} / \mathrm{s}$, and $n$ and $B$ are taken from equations 8 and 9 . The circles and triangles correspond to observed kinetic temperatures for $\mathrm{O}^{+5}$ ions and protons from Esser et al. (1999), with the error bars included in the left panel only.

protons can be obtained by comparing the cascade power $\Gamma$ with the heating rate $Q_{\mathrm{p}, \text { crit }}=k_{\mathrm{B}} T_{\perp \mathrm{p}} /\left(m_{\mathrm{p}} t_{\mathrm{exp}}\right)$ required to sustain protons at the temperature $T_{\perp p}$ given by equations $(19)$ and (20). If the condition $Q_{\mathrm{p} \text {,crit }}<\Gamma$ were not satisfied, then protons would absorb so much energy from the cascade that $\alpha_{p}$ would drop below the assumed value of 0.71 , thereby reducing $Q_{\mathrm{p}, \text { crit }}$ below $\Gamma$ as required by energy conservation. The value of $Q_{\mathrm{p}, \text { crit }} / \Gamma$ is shown in figure 4 for $1.8 R_{\odot}<r<$ $15 R_{\odot}$ for the same set of assumptions used to obtain the proton temperature profile in figure 3 It can be seen that $Q_{\mathrm{p}, \text { crit }}<\Gamma$ throughout this range of $r$. However, some caution is warranted here. As noted by Chandran \& Hollweg (2009), equation (6) likely overestimates the turbulent dissipation rate for two reasons. First, large-scale Alfvén waves launched from the Sun must propagate some distance into the corona before their energy cascades all the way to the dissipation scale. Second, equation (6) is derived in the limit of small $L_{\perp}$. As shown in figure 3 of Chandran \& Hollweg (2009) (and in figure 5 of Dmitruk et al. (2002) for the case $U=0$ ), finite values of $L_{\perp}$ reduce $\Gamma$ relative to the small- $L_{\perp}$ limit. These effects are particularly important close to the Sun. The small value of $Q_{\text {p.crit }} / \Gamma$ at $r \sim 2 R_{\odot}$ shown in figure 4 may thus significantly underestimate the fraction of the cascade power absorbed by protons at this location.

\section{CONCLUSION}

Low-frequency Alfvén-wave/KAW turbulence offers a promising explanation for the detailed features of ion kinetic temperatures seen in UVCS observations of coronal holes, including the abrupt radial variations in the $\mathrm{O}^{+5}$ temperature profile, the widely different temperatures of protons and $\mathrm{O}^{+5}$ ions, and the large $\mathrm{O}^{+5}$ temperature anisotropy $\left(T_{\perp \mathrm{i}} \gg\right.$ $T_{\| \mathrm{i}}$ ). When $\beta \ll 1$, ion heating from the linear damping of low-frequency Alfvén waves and KAWs can be neglected,

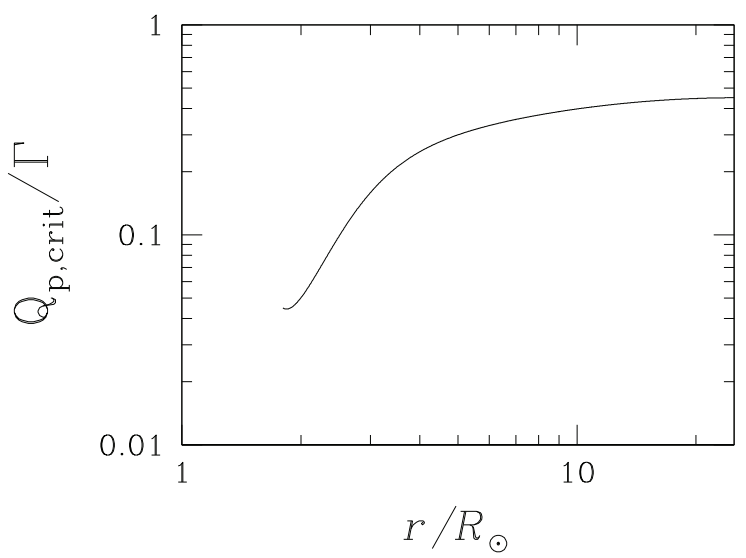

FIG. 4.- $Q_{\mathrm{p} \text {,crit }}$ is the approximate heating rate required to sustain protons at the temperature in the right panel of figure 3 given the outflow speed in equation (10). $\Gamma$ is the turbulent dissipation rate in equation (6).

and stochastic heating is arguably the primary way in which low-frequency Alfvén-wave/KAW turbulence heats ions. As shown by Chandran et al. (2010), the stochastic heating rate is a strongly decreasing function of $T_{\perp \mathrm{i}}$. At small $v_{\perp \mathrm{i}}$, $\varepsilon_{\mathrm{i}}$ is comparatively large and ion gyro orbits are strongly perturbed as the ions traverse the electrostatic potential of the gyroscale fluctuations. This leads to chaotic ion orbits and strong stochastic heating. In contrast, at large $v_{\perp \mathrm{i}}$, ion gyromotion is only weakly perturbed by the electrostatic potential of the turbulent fluctuations, ions drift smoothly with nearly circular orbits in the plane perpendicular to $\mathbf{B}$, and stochastic heating is weak. As discussed in section 4 the observed $\mathrm{O}^{+5}$ temperature increases rapidly from $\sim 10^{7} \mathrm{~K}$ to $\sim 10^{8} \mathrm{~K}$ between $r=1.6 R_{\odot}$ and $r=1.9 R_{\odot}$, as the ions leave the region at 
$r \lesssim 1.6 R_{\odot}$ in which collisional energy exchange with protons is important (Esser et al. 1999). At $r=1.9 R_{\odot}$, the $\mathrm{O}^{+5}$ temperature profile abruptly flattens, and $T_{\perp \mathrm{i}}$ remains $\sim 10^{8} \mathrm{~K}$ out to $2.7 R_{\odot}$, the largest radius observed. This temperature profile is consistent with rapid stochastic heating of $\mathrm{O}^{+5}$ at $T_{\perp \mathrm{i}} \sim 10^{7} \mathrm{~K}$ that saturates at $T_{\perp \mathrm{i}} \sim 10^{8} \mathrm{~K}$ due to the reduction in orbit stochasticity.

Ion thermal conduction, like collisional energy exchange between particle species, can be neglected to a good approximation at $r \gtrsim 2 R_{\odot}$. As a result, the strong temperature dependence of the heating rate causes all minor ion temperatures to evolve at $r \gtrsim 2 R_{\odot}$ to values at which $t_{\mathrm{h}} \sim t_{\exp }$; at lower $T_{\perp \mathrm{i}}$, minor ions are rapidly heated, while at higher temperatures heating is ineffective and ions cool adiabatically. Because of the exponential dependence of $t_{\mathrm{h}}$ on $\varepsilon_{\mathrm{i}}$, the condition $t_{\mathrm{h}} \sim t_{\exp }$ is satisfied by different ion species at nearly equal values of $\varepsilon_{\mathrm{i}}$. This then leads to slightly more than massproportional temperatures - in particular, $T_{\perp \mathrm{i}} \propto A \cdot(A / Z)^{l}$ for ions with the same values of $\varepsilon_{i}$ and $\alpha_{i}$, as in equations (20) and (21). The proton temperature is even smaller relative to minor-ion temperatures than the scaling in equation (20) with constant $\alpha_{i}$ would suggest, because proton heating and electron Landau damping reduce the amplitude of the fluctuations at $\lambda_{\perp} \sim \rho_{\mathrm{p}}$, which make the largest contribution to the stochastic proton heating rate. The condition $t_{\mathrm{h}} \sim t_{\mathrm{exp}}$ thus leads to $\mathrm{O}^{+5}$ temperatures that are much higher than the proton temperature, consistent with UVCS observations. As described briefly in section 2, stochastic heating primarily increases the speed of ion thermal motions perpendicular to $\mathbf{B}$ when $\beta \ll 1$ (Chandran et al. 2010). Because of this, stochastic heating also offers an explanation for the observation that $T_{\perp \mathrm{i}} \gg T_{\| \mathrm{i}}$ for $\mathrm{O}^{+5}$ ions Kohl, J.. et al. 1998; Li et al. 1998; Antonucci et al. 2000).

In section 4, $T_{\perp \mathrm{i}}$ profiles are calculated for several ion species from the condition $t_{\mathrm{h}} \sim t_{\exp }$ using an observationally constrained model of Alfvén-wave turbulence in coronal holes. For plausible values of the model parameters, the resulting temperature profiles provide a good match to obser- vations of protons and $\mathrm{O}^{+5}$ ions. However, there are several sources of uncertainty in these calculations, the largest of which are associated with the parameters $c_{2}, a$, and $\alpha_{\mathrm{p}}$ (the value of $\alpha_{i}$ for protons). The constant $c_{2}$ relates to the efficiency of stochastic ion heating and was evaluated by Chandran et al. (2010) using numerical simulations of testparticle protons interacting with randomly phased waves in a low- $\beta$ plasma. However, the value of $c_{2}$ for strong turbulence and for other ion species is not yet known. The quantities $a$ and $\alpha_{p}$ describe, respectively, the slope of the inertial-range velocity power spectrum and the decrement in the velocity spectrum at $\lambda_{\perp} \sim \rho_{\mathrm{p}}$ arising from electron and ion damping. In order to determine whether stochastic heating can indeed explain the ion temperatures observed in coronal holes, future work is needed to determine $c_{2}, a$, and $\alpha_{\mathrm{p}}$ more accurately. For example, direct numerical simulations or a cascade model (see, e.g., Howes et al. (2008a)) of strong AW/KAW turbulence including both stochastic heating and electron Landau damping would help ascertain the value of $\alpha_{p}$. Direct numerical simulations of strong, highly anisotropic, AW/KAW turbulence interacting with test particles could be used to determine the value of $c_{2}$ that is appropriate for coronal holes. In addition, in situ measurements from NASA's planned Solar Probe mission will establish the power spectrum of AW/KAW turbulence and determine $U_{\mathrm{i}}(r)$ and $T_{\perp \mathrm{i}}(r)$ for several ion species at heliocentric distances as small as $\sim 9.5 R_{\odot}$. These measurements will lead to a rigorous test of the stochastic heating model, including the $T_{\perp \mathrm{i}}(r)$ predictions shown in figure 3 and the dependence of $T_{\perp \mathrm{i}}$ on ion mass and charge given in equation (21).

I thank Joe Hollweg, Phil Isenberg, Eliot Quataert, and the referee for valuable suggestions and feedback on an earlier version of this manuscript, and Peter Bochsler, Bo Li, and Barrett Rogers for helpful discussions. This work was supported in part by NSF Grants AST-0613622, AGS-0851005, and AGS-1003451, DOE Grant DE-FG02-07-ER46372, and NASA Grants NNX07AP65G and NNX08AH52G.

\section{REFERENCES}

Antonucci, E., Dodero, M. A., \& Giordano, S. 2000, Sol. Phys., 197, 115 Bale, S. D., Kellogg, P. J., Mozer, F. S., Horbury, T. S., \& Reme, H. 2005, Physical Review Letters, 94, 215002

Bame, S. J., Asbridge, J. R., Feldman, W. C., \& Gosling, J. T. 1977, J. Geophys. Res., 82, 1487

Belcher, J. W., \& Davis, Jr., L. 1971, J. Geophys. Res., 76, 3534

Boldyrev, S. 2006, Physical Review Letters, 96, 115002

Bourouaine, S., Marsch, E., \& Vocks, C. 2008, ApJ, 684, L119

Bruno, R., \& Carbone, V. 2005, Living Reviews in Solar Physics, 2, 4

Chandran, B. D. G., \& Hollweg, J. V. 2009, ApJ, 707, 1659

Chandran, B. D. G., Li, B., Rogers, B. N., Quataert, E., \& Germaschewski, K. 2010, ApJ, submitted, (arXiv:1001.2069)

Chandran, B. D. G., Quataert, E., Howes, G. G., Xia, Q., \&

Pongkitiwanichakul, P. 2009, ApJ, 707, 1668

Chen, L., Lin, Z., \& White, R. 2001, Physics of Plasmas, 8, 4713

Cho, J., Lazarian, A., \& Vishniac, E. T. 2002, ApJ, 564, 291

Cho, J., \& Vishniac, E. T. 2000, ApJ, 539, 273

Coleman, P. J. 1968, ApJ, 153, 371

Cranmer, S. R. 2010, ApJ, 710, 676

Cranmer, S. R., Field, G. B., \& Kohl, J. L. 1999, ApJ, 518, 937

Cranmer, S. R., Matthaeus, W. H., Breech, B. A., \& Kasper, J. C. 2009, ApJ, 702,1604

Cranmer, S. R., \& van Ballegooijen, A. A. 2003, ApJ, 594, 573

-. 2005, 156, 265

Cranmer, S. R., van Ballegooijen, A. A., \& Edgar, R. J. 2007, ApJS, 171, 520

De Pontieu, B., et al. 2007, Science, 318, 1574
Dmitruk, P., Matthaeus, W. H., Milano, L. J., Oughton, S., Zank, G. P., \& Mullan, D. J. 2002, ApJ, 575, 571

Dmitruk, P., Matthaeus, W. H., \& Seenu, N. 2004, ApJ, 617, 667

Drake, J. F., Cassak, P. A., Shay, M. A., Swisdak, M., \& Quataert, E. 2009, ApJ, 700, L16

Esser, R., Fineschi, S., Dobrzycka, D., Habbal, S. R., Edgar, R. J., Raymond, J. C., Kohl, J. L., \& Guhathakurta, M. 1999, ApJ, 510, L63

Feldman, W. C., Habbal, S. R., Hoogeveen, G., \& Wang, Y. 1997, J. Geophys. Res., 102, 26905

Galtier, S., Nazarenko, S. V., Newell, A. C., \& Pouquet, A. 2000, Journal of Plasma Physics, 63, 447

Goldreich, P., \& Sridhar, S. 1995, ApJ, 438, 763

Goldstein, M. L., Roberts, D. A., \& Matthaeus, W. H. 1995, ARA\&A, 33, 283

Grappin, R., Mangeney, A., \& Marsch, E. 1990, J. Geophys. Res., 95, 8197

Harmon, J. K., \& Coles, W. A. 2005, Journal of Geophysical Research (Space Physics), 110, 3101

Haugen, N. E., Brandenburg, A., \& Dobler, W. 2004, Phys. Rev. E, 70, 016308

Heinemann, M., \& Olbert, S. 1980, J. Geophys. Res., 85, 1311

Hollweg, J. V. 1999, J. Geophys. Res., 104, 14811

Hollweg, J. V., \& Isenberg, P. A. 2007, Journal of Geophysical Research (Space Physics), 112, 8102

Howes, G. G., Cowley, S. C., Dorland, W., Hammett, G. W., Quataert, E., \& Schekochihin, A. A. 2008a, Journal of Geophysical Research (Space Physics), 113, 5103 
Howes, G. G., Dorland, W., Cowley, S. C., Hammett, G. W., Quataert, E., Schekochihin, A. A., \& Tatsuno, T. 2008b, Physical Review Letters, 100, 065004

Isenberg, P. A., \& Vasquez, B. J. 2009, ApJ, 696, 591

Johnson, J. R., \& Cheng, C. Z. 2001, Geophys. Res. Lett., 28, 4421

Kasper, J. C., Stevens, M. L., Lazarus, A. J., Steinberg, J. T., \& Ogilvie, K. W. 2007, ApJ, 660, 901

Kohl, J., et al. 1998, ApJ, 501, L127

Kruskal, M. 1962, Journal of Mathematical Physics, 3, 806

Leamon, R. J., Smith, C. W., Ness, N. F., \& Wong, H. K. 1999, J. Geophys. Res., 104, 22331

Lehe, R., Parrish, I. J., \& Quataert, E. 2009, ApJ, 707, 404

Li, X., Habbal, S. R., Kohl, J., \& Noci, G. 1998, ApJ, 501, L133+

Markovskii, S. A., Vasquez, B. J., Smith, C. W., \& Hollweg, J. V. 2006, ApJ, 639, 1177

Maron, J., \& Goldreich, P. 2001, ApJ, 554, 1175

Marsch, E., Ao, X.-Z., \& Tu, C.-Y. 2004, Journal of Geophysical Research (Space Physics), 109, 4102

Marsch, E., Rosenbauer, H., Schwenn, R., Muehlhaeuser, K., \& Neubauer, F. M. 1982, J. Geophys. Res., 87, 35

Mason, J., Cattaneo, F., \& Boldyrev, S. 2008, Phys. Rev. E, 77, 036403

Matthaeus, W. H., Zank, G. P., Oughton, S., Mullan, D. J., \& Dmitruk, P. 1999, ApJ, 523, L93

Müller, W., \& Grappin, R. 2005, Physical Review Letters, 95, 114502

Müller, W.-C., \& Biskamp, D. 2000, Physical Review Letters, 84, 475

Ng, C. S., \& Bhattacharjee, A. 1996, ApJ, 465, 845

Parashar, T. N., Shay, M. A., Cassak, P. A., \& Matthaeus, W. H. 2009, Physics of Plasmas, 16, 032310

Perez, J. C., \& Boldyrev, S. 2008, ApJ, 672, L61
—. 2009, Physical Review Letters, 102, 025003

Phillips, J. L., \& Gosling, J. T. 1990, J. Geophys. Res., 95, 4217

Podesta, J. J., Roberts, D. A., \& Goldstein, M. L. 2007, ApJ, 664, 543

Quataert, E. 1998, ApJ, 500, 978

Sahraoui, F., Goldstein, M. L., Robert, P., \& Khotyaintsev, Y. V. 2009,

Physical Review Letters, 102, 231102

Schekochihin, A. A., Cowley, S. C., Dorland, W., Hammett, G. W., Howes, G. G., Quataert, E., \& Tatsuno, T. 2009, ApJS, 182, 310

Shebalin, J. V., Matthaeus, W., \& Montgomery, D. 1983, Journal of Plasma Physics, 29, 525

Smith, C. W., Matthaeus, W. H., Zank, G. P., Ness, N. F., Oughton, S., \& Richardson, J. D. 2001, J. Geophys. Res., 106, 8253

Spruit, H. C. 1981, NASA Special Publication, 450, 385

Stawarz, J. E., Smith, C. W., Vasquez, B. J., Forman, M. A., \& MacBride, B. T. 2009, ApJ, 697, 1119

Tomczyk, S., McIntosh, S. W., Keil, S. L., Judge, P. G., Schad, T., Seeley,

D. H., \& Edmondson, J. 2007, Science, 317, 1192

Tu, C., \& Marsch, E. 1995, Space Science Reviews, 73, 1

Velli, M. 1993, A\&A, 270, 304

Velli, M., Grappin, R., \& Mangeney, A. 1989, Physical Review Letters, 63, 1807

Verdini, A., \& Velli, M. 2007, ApJ, 662, 669

Verdini, A., Velli, M., Matthaeus, W. H., Oughton, S., \& Dmitruk, P. 2010, ApJ, 708, L116

Voitenko, Y., \& Goossens, M. 2004, ApJ, 605, L149

White, R., Chen, L., \& Lin, Z. 2002, Physics of Plasmas, 9, 1890 